\title{
Dihedral F-Tilings of the Sphere by Equilateral and Scalene Triangles-I
}

\author{
A. M. d’Azevedo Breda (*) - Patrícia S. Ribeiro(**) \\ Altino F. SANTos (***)
}

ABSTRACT - The study of dihedral f-tilings of the Euclidean sphere $S^{2}$ by triangles and $r$-sided regular polygons was initiated in 2004 where the case $r=4$ was considered [6]. In a subsequent paper [1], the study of all spherical ftilings by triangles and $r$-sided regular polygons, for any $r \geq 5$, was described. Later on, in [3] the classification of all f-tilings of $S^{2}$ whose prototiles are an equilateral triangle (a 3 -sided regular polygon) and an isosceles triangle are obtained. In this paper we extend these results presenting the study of all spherical f-tilings by equilateral triangles and scalene triangles in a particular way of adjacency. For this particular case of adjacency, we get two continuous families of f-tilings denoted by $\mathcal{F}_{1}^{\delta}$ and $\mathcal{F}_{2}^{\delta}$. The combinatorial structure including the transitive properties under the action of their symmetry groups is given in Table 1.

\section{Introduction}

Spherical folding tilings or f-tilings for short, are edge-to-edge decompositions of the sphere by geodesic polygons, such that all vertices are of even valency and the sums of alternate angles around each vertex are $\pi$, i.e., each vertex is of even valency $2 n, n \geq 2$ and the sums of alternate

(*) Indirizzo dell'A.: Department of Mathematics, University of Aveiro, 3810-193 Aveiro, Portugal.

E-mail: ambreda@mat.ua.pt

(**) Indirizzo dell'A.: Department of Mathematics, E.S.T. Setúbal, 2910-761, Setúbal, Portugal.

E-mail: pribeiro@est.ips.pt

(***) Indirizzo dell'A.: Department of Mathematics, U.T.A.D., 5001-801, Vila

Real, Portugal.

E-mail: afolgado@utad.pt

2000 Mathematics Subject Classification: 52C20, 52B05, $20 \mathrm{~B} 35$. 
angles are equal, that is

$$
\sum_{i=1}^{n} \alpha_{2 i}=\sum_{i=1}^{n} \alpha_{2 i-1}=\pi
$$

where the angles $\alpha_{i}$ around any vertex are ordered cyclically. A f-tiling $\tau$ is said to be monohedral if it is composed by congruent cells, and dihedral if every tile of $\tau$ is congruent to one of two fixed sets $X$ and $Y$ (prototiles of $\tau$ ). We shall denote by $\Omega(X, Y)$ the set, up to isomorphism, of all dihedral ftilings of $S^{2}$ whose prototiles are $X$ and $Y$.

Spherical f-tilings are strongly related to the theory of isometric foldings [9]. The study of this special class of tilings was initiated in [2] with a complete classification of all spherical monohedral f-tilings. The classification of all spherical dihedral folding tilings by rhombi and triangles was recently obtained in 2005, [7].

Here, our interest is focused in spherical triangular dihedral f-tilings whose prototiles are an equilateral triangle and a scalene triangle, in a particular way of adjacency (see I in Figure 1). We end up with two continuous families of f-tilings, illustrated in Figures 18 and 19. The other two cases of adjacency were studied in [4] and [5]. With this study the classification of the spherical f-tilings by two triangular tiles one of which equilateral is now completed.

From now on $T_{1}$ denotes an equilateral spherical triangle of angle $\alpha$ and side $a$ and $T_{2}$ a scalene spherical triangle of angles $\delta, \gamma, \beta$, with the order relation $\delta<\gamma<\beta$ and with sides $b$ (opposite to $\beta$ ), $c$ (opposite to $\gamma$ ) and $d$ (opposite to $\delta$ ).

We begin by pointing out that any element of $\Omega\left(T_{1}, T_{2}\right)$ has at least two cells congruent, respectively, to $T_{1}$ and $T_{2}$, such that they are in adjacent positions in one and only one of the situations illustrated in Figure 1.

The type I edge-adjacency condition can be analytically described by the equation (see [8])

$$
\frac{\cos \alpha(1+\cos \alpha)}{\sin ^{2} \alpha}=\frac{\cos \beta+\cos \delta \cos \gamma}{\sin \delta \sin \gamma} .
$$
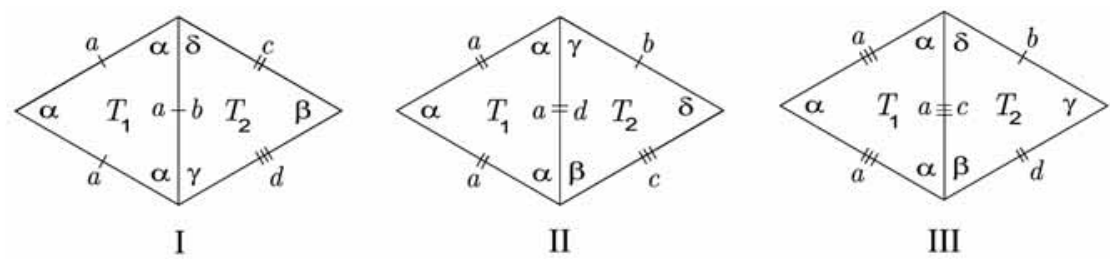

Fig. 1. - Distinct cases of adjacency. 
In order to get any dihedral f-tiling $\tau \in \Omega\left(T_{1}, T_{2}\right)$, we find useful to start by considering one of its planar representations, beginning with a common vertex to an equilateral triangle and a scalene triangle in adjacent positions. In the diagrams that follows it is convenient to label the tiles according to the following procedures:

(i) The tiles by which we begin the planar representation of a tiling $\tau \in \Omega\left(T_{1}, T_{2}\right)$ are labelled by 1 and 2 , respectively;

(ii) For $j \geq 2$, the location of tile $j$ can be deduced from the configuration of tiles $(1,2, \ldots, j-1)$ and from the hypothesis that the configuration is part of a complete planar representation of a f-tiling (except in the cases indicated).

\section{Triangular Dihedral F-Tilings with Adjacency of Type I.}

Let $T_{1}$ and $T_{2}$ be tiles with adjacency of type I.

Starting a planar representation of a such tiling $\tau \in \Omega\left(T_{1}, T_{2}\right)$ we are led to the one illustrated in Figure 2, where $x \in\{\beta, \gamma\}$.

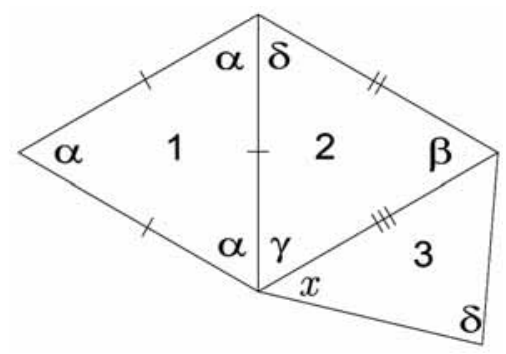

Fig. 2. - Planar representation.

The cases $x=\beta$ and $x=\gamma$ will be studied separately.

Let us begin by assuming that $x=\beta$. Accordingly, $\alpha+\beta \leq \pi$.

Proposition 2.1. If $T_{1}$ and $T_{2}$ are tiles with adjacency of type $I$ and $\alpha+x \leq \pi$, with $x=\beta$, then $\Omega\left(T_{1}, T_{2}\right)=\varnothing$.

Proof. Suppose that $\alpha+x=\pi$, with $x=\beta$. The configuration of $\tau$ illustrated in Figure 2 expands to the following one (Figure 3).

Since $\alpha+\beta=\gamma+\delta=\pi$ and $\beta>\gamma$, then $\alpha<\delta$. Therefore, $\alpha<\delta<\frac{\pi}{2}<\gamma<\beta$. Accordingly, $\cos \alpha, \cos \delta>0$ while $\cos \beta, \cos \gamma<0$ contradicting the adjacency condition 1.1. 


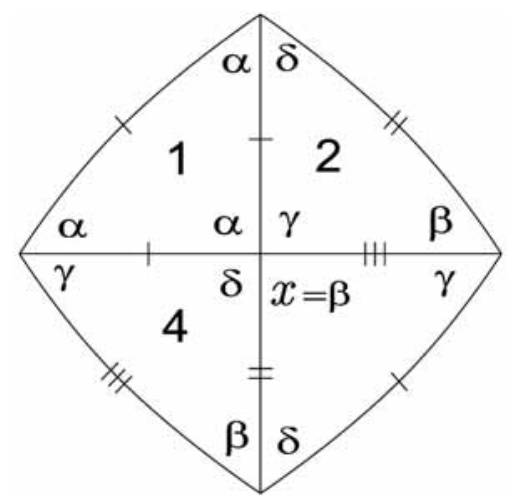

Fig. 3. - Planar representation.

If $\alpha+\beta<\pi$ the edge compatibility around a vertex surrounded by a sequence of angles of type $(\star, \star, \ldots, \alpha, \star, \beta, \star, \ldots)$ implies that all the angles, in this sequence, different from $\alpha$ and $\beta$ must have amplitude $\delta$. Consequently, $\alpha+\beta+k \delta=\pi$, for some integer $k \geq 1$.

The configuration shown in Figure 2 can be expanded to the one illustrated in Figure 4.

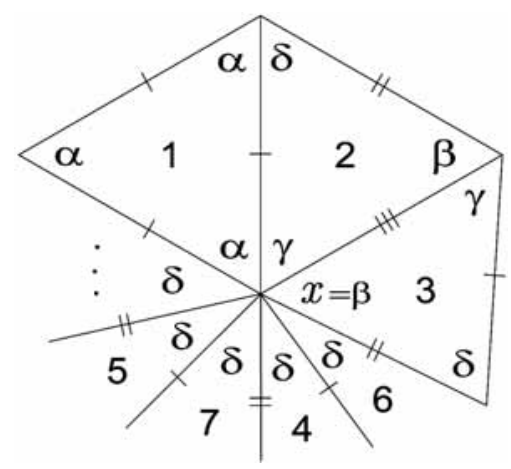

Fig. 4. - Planar representation.

In this situation we end up with the equation $\alpha+\beta+k \delta=\pi=\gamma+(k+1) \delta$ from which we derive the following condition $\alpha+\beta=\gamma+\delta$ and consequently $\frac{\pi}{3}<\alpha<\delta<\gamma<\beta$, contradicting $\alpha+\beta+k \delta=\pi$, for some integer $k \geq 1$.

Let us assume now that $x=\gamma$, see Figure 2 . 


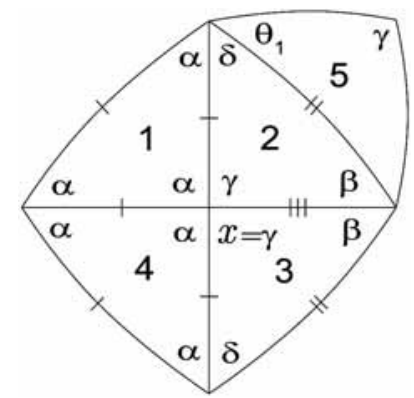

Fig. 5. - Planar representation.

Proposition 2.2. If $T_{1}$ and $T_{2}$ are tiles with adjacency of type $I$ and $\alpha+\gamma \leq \pi$, then $\Omega\left(T_{1}, T_{2}\right) \neq \varnothing$ if and only if $\beta=\frac{\pi}{2}$ and $\alpha+\gamma+\delta=\pi$ ending up with two non isomorphic dihedral $f$-tilings.

Proof. A) If $\alpha+\gamma=\pi$, the configuration in Figure 2 expands to the one illustrated in Figure 5.

A decision about the angle $\theta_{1} \in\{\delta, \beta\}$ must be taken. Having in account that $\pi=\alpha+\gamma<\alpha+\beta$, then $\theta_{1}=\delta$ (Figure 6).

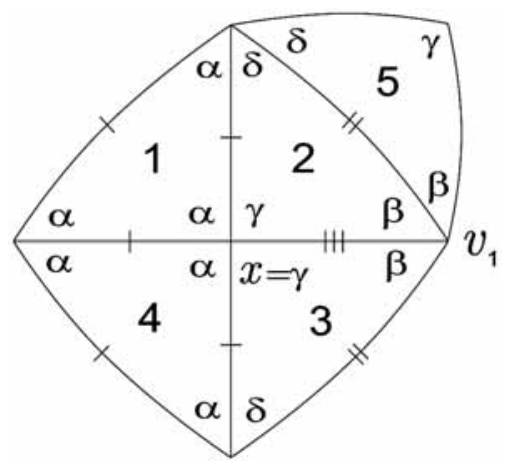

Fig. 6. - Planar representation.

One of the alternate angle sum at vertex $v_{1}$ is $2 \beta \leq \pi$. If $\beta<\frac{\pi}{2}$ one has $\delta<\gamma<\frac{\pi}{2}$ and $\alpha>\frac{\pi}{2}$, violating the adjacency condition 1.1. Accordingly $\beta=\frac{\pi}{2}$.

B) Consider now that $\alpha+\gamma<\pi$. The angle $\theta_{2}$ belongs to the set $\{\delta, \beta\}$, see Figure 7. 


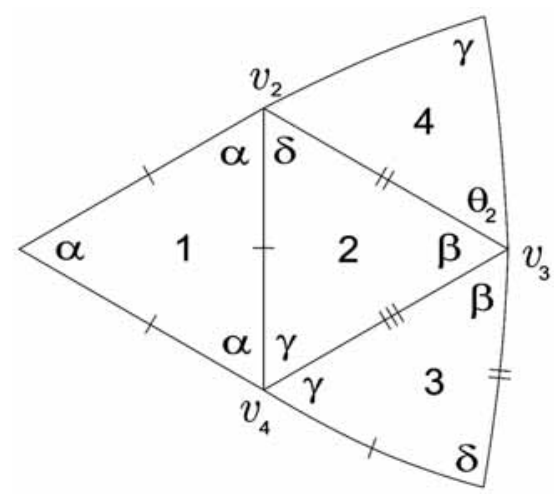

Fig. 7. - Planar representation.

1. Suppose, firstly, that $\theta_{2}=\delta$.

The alternate angle sum at vertex $v_{2}$ containing $\alpha$ and $\beta$ satisfies $\alpha+\beta<\pi$, otherwise it would become $\alpha+\beta=\pi=\delta+\gamma$. Therefore, we would have $\alpha<\delta<\frac{\pi}{2}<\gamma<\beta$, not satisfying the adjacency condition 1.1. As $\alpha+\beta<\pi$ and having in account that $\alpha, \beta>\frac{\pi}{3}$ and $\alpha+\beta+\gamma>\pi$ then $\alpha+\beta+k \delta=\pi$, for some $k \geq 1$ (vertex $v_{2}$ ). Now the alternate angle sum containing $\beta$ and $\delta$ at vertex $v_{3}$ satisfies $\beta+\delta<\pi$. Summarizing, since $\beta+\gamma+\delta>\pi$ and $\alpha+\beta+k \delta=\pi, k \geq 1$, then $\delta<\frac{\pi}{3}<\alpha<\gamma<\beta$.

Consequently, the alternate angle sum, at vertex $v_{4}$, containing $\alpha$ and $\gamma$ is $\alpha+\gamma+t \delta=\pi$, for some $t>k \geq 1$. The configuration extends to the one in Figure 8.

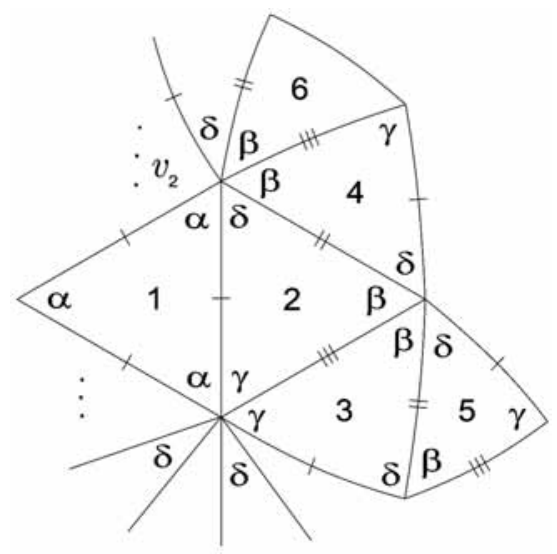

Fig. 8. - Planar representation. 


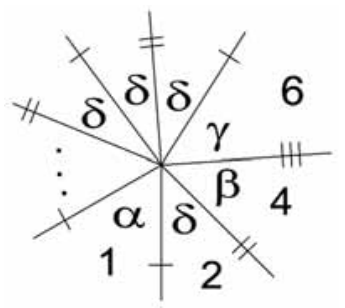

Fig. 9. - Angles arrangement around vertex $v_{2}$.

Observe that in tile numbered 6 , if the angle $\beta$ was $\gamma$, the vertex $v_{2}$ would be surrounded by the sequence of angles illustrated in Figure 9 . The alternate angle sums would be $\alpha+\beta+k \delta=\pi=\delta+\gamma+k \delta$. Therefore, $\alpha+\beta=\delta+\gamma$, which is absurd since $\delta<\alpha<\gamma<\beta$.

We may add some new cells to the configuration in Figure 8 and we get the one illustrated in Figure 10.

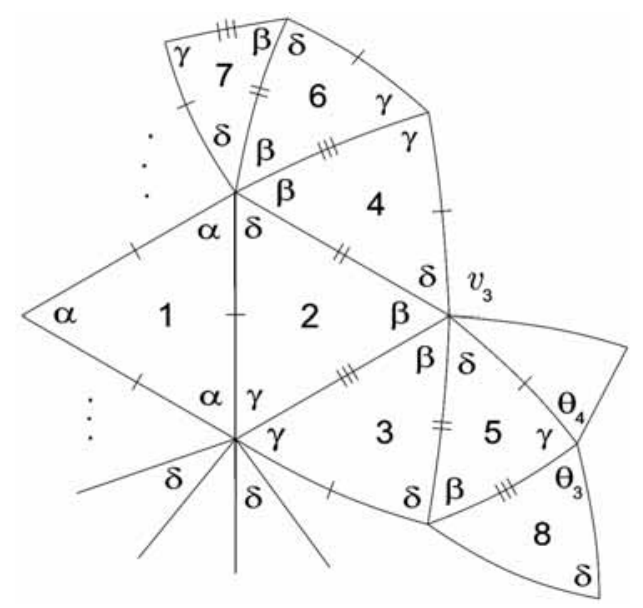

Fig. 10. - Planar representation.

1.1. If $\theta_{3}=\beta$, then $\theta_{4}=\gamma$. Observe that $\theta_{4}=\alpha$ leads to the cyclic sequence $(\alpha, \gamma, \beta, \ldots)$, which is absurd (case already studied in the previous proposition). Thus, the alternate angle sums at vertex $v_{3}$ are $\beta+r \delta=\pi$, for some $r \geq 1$. Consequently, at vertex $v_{5}$ (Figure 11) we have $\beta+\gamma=\pi=2 \gamma$ getting again a contradiction, since $\gamma<\beta$. 


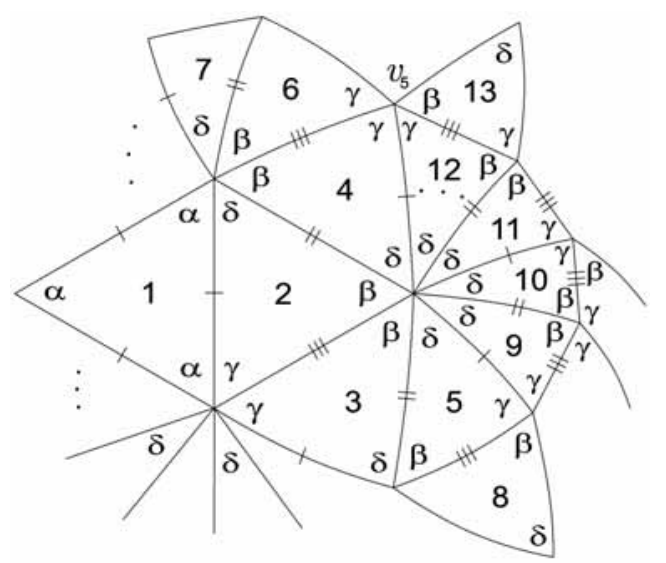

Fig. 11. - Planar representation.

1.2 If $\theta_{3}=\gamma$, the configuration in Figure 10 gives rise to the one in Figure 12.

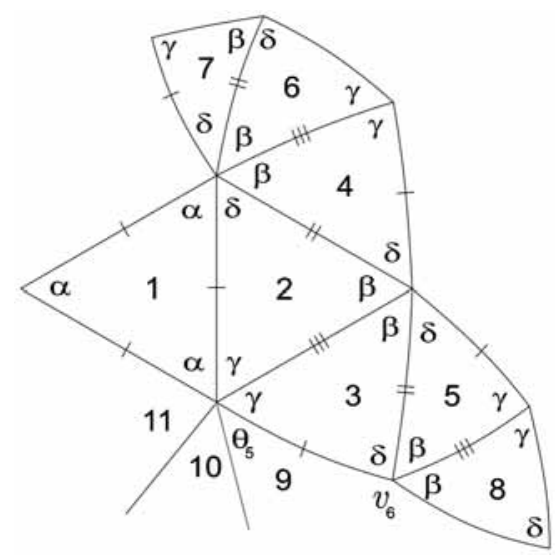

Fig. 12. - Planar representation.

Now, $\theta_{5}$ is different from $\delta$, otherwise, at vertex $v_{6}$ we get the cyclic sequence $(\gamma, \delta, \beta, \beta)$ and the alternate angle sums would be $\beta+\gamma=\pi=\beta+\delta$, which is an absurd. Due to the compatibility of sides in tiles 10 and 11, $\theta_{5}$ must be different from $\gamma$. Therefore, $\theta_{5}=\alpha$ and extending the previous configuration, we conclude that $\beta=\frac{\pi}{2}$, see Figure 13. 


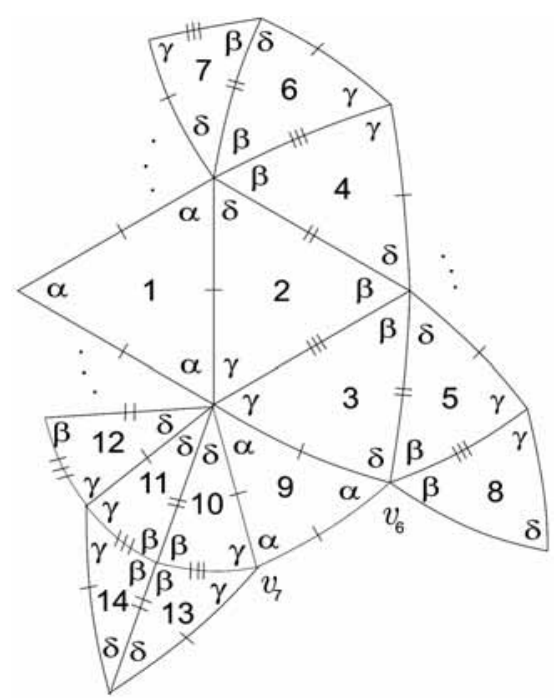

Fig. 13. - Planar representation.

Consequently $\gamma+\delta>\frac{\pi}{2}$ and $\gamma<\frac{\pi}{2}$. Accordingly, $\delta<\frac{\pi}{3}<\alpha<\gamma<\frac{\pi}{2}=\beta$ and all the alternate angle sums containing two angles $\gamma$ are $2 \gamma+\delta=\pi$. Having in account that, $\beta=\frac{\pi}{2}, 2 \gamma+\delta=\pi, \alpha+\gamma+t \delta=\pi$ and $\alpha+\beta+k \delta=\pi$, one has $\alpha+k \delta=\frac{\pi}{2}$ and $(1-2 t-2 k) \delta=0$, which is absurd since $t>k \geq 1$.

2. Suppose now that $\theta_{2}=\beta$ (see Figure 7). Then, $\beta=\frac{\pi}{2}$ and $\alpha<\frac{\pi}{2}$ (otherwise the adjacency condition 1.1 is violated, since $\delta<\gamma<\frac{\pi}{2}$ ). Also

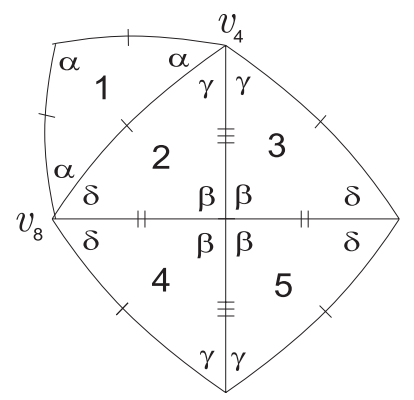

Fig. 14. - Planar representation. 
$\delta<\alpha$, otherwise $\delta>\frac{\pi}{3}$ and $\alpha+\delta+\rho>\pi$, for any $\rho \in\{\alpha, \delta, \gamma, \beta\}$. Looking at vertex $v_{4}$ in Figure 7, the alternate angle sum containing $\alpha$ and $\gamma$ is $\alpha+\gamma+\alpha=\pi$ or $\alpha+\gamma+\gamma=\pi$ or $\alpha+\gamma+t \delta=\pi$, for some $t \geq 1$, see Figure 14 .

2.1 If $\alpha+\gamma+\alpha=\pi$, then $\frac{\pi}{6}<\delta<\gamma<\frac{\pi}{3}<\alpha$. At vertex $v_{8}$, the alternate angle sum containing $\alpha$ and $\delta$ must be

$$
2 \alpha+\delta=\pi \quad \text { or } \quad \alpha+\delta+\gamma=\pi \quad \text { or } \quad \alpha+2 \delta+\gamma=\pi \quad \text { or } \quad \alpha+3 \delta=\pi .
$$

Clearly, the first two cases are impossible. Therefore, $\alpha+2 \delta+\gamma=\pi$ or $\alpha+3 \delta=\pi$. As $\delta+\gamma>\frac{\pi}{2}$ and $\alpha>\gamma$, then $\alpha+2 \delta+\gamma>\pi$ and so $\alpha+3 \delta=\pi$, that is $\delta<\frac{2 \pi}{9}$. The angular sequence around vertex $v_{8}$ of valency 8 , is of the form $(\alpha, \alpha, \delta, \ldots, \delta)$ or $(\alpha, \delta, \delta, \alpha, \delta, \ldots)$.

Either way, in the expanded configuration, having in account the edge compatibility we get a vertex surrounded by an angular sequence containing four consecutive $\gamma$ angles, as illustrated in Figure 15. As $\gamma+\delta>\frac{\pi}{2}$ and $\delta<\gamma<\alpha$ then, $2 \gamma+\delta<2 \gamma+\gamma<\pi, 2 \gamma+2 \gamma>2 \gamma+2 \delta>\pi, 2 \gamma+\alpha<\gamma+2 \alpha=\pi$ and $3 \gamma+\alpha>3 \gamma+\gamma>\pi$. Consequently it is impossible to continue extending the configuration.

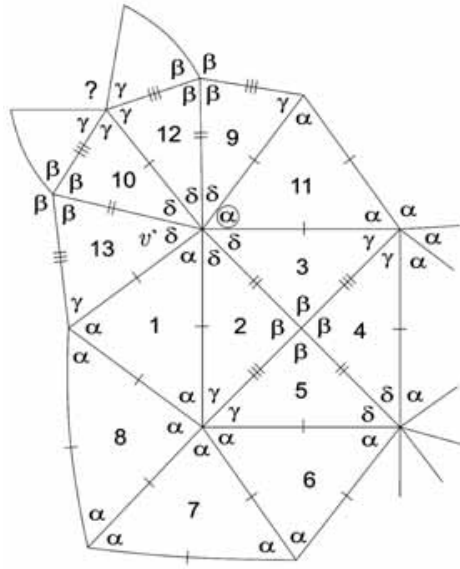

I

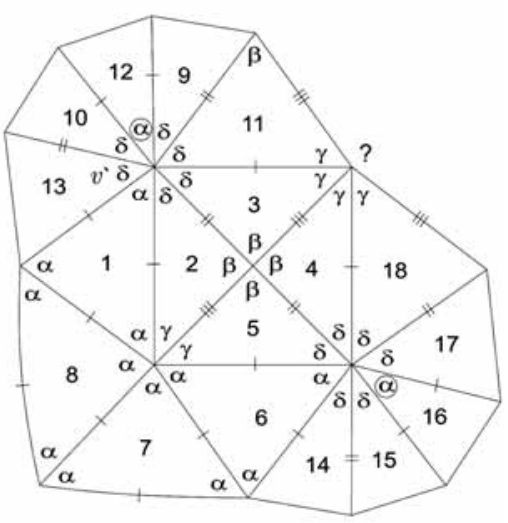

II

Fig. 15. - Planar representations. 
2.2 If $\alpha+2 \gamma=\pi$, the angular order relation is exactly the same,

$$
\frac{\pi}{6}<\delta<\gamma<\frac{\pi}{3}<\alpha
$$

The alternate angle sum at vertex $v_{8}$ containing $\alpha$ and $\delta$ must be

$\alpha+\delta+\gamma=\pi \quad$ or $\quad \alpha+2 \delta+\gamma=\pi \quad$ or $\quad \alpha+3 \delta=\pi, \quad$ or $\quad 2 \alpha+\delta=\pi$.

The first case is incompatible with the assumption $\alpha+2 \gamma=\pi$.

As $\gamma+\delta>\frac{\pi}{2}$ and $\alpha>\gamma$, then $\alpha+2 \delta+\gamma>\pi$ and the second case must be eliminated. The third case should be discarded, using a similar reasoning to the one made in case 2.1 under the same assumption. Suppose now that $2 \alpha+\delta=\pi$. By the adjacency condition we get $\gamma \approx 56.489^{\circ}, \alpha \approx 67.022^{\circ}$ and $\delta \approx 45.956^{\circ}$.

Starting from configuration in Figure 14 we are led, according to the edge position of tile 6 , to one of the configurations illustrated in Figure 16.

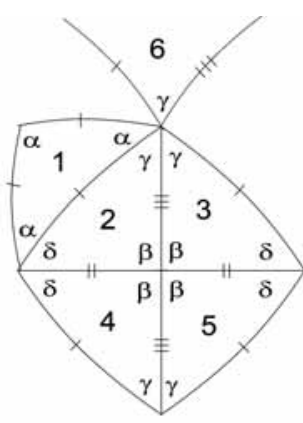

I

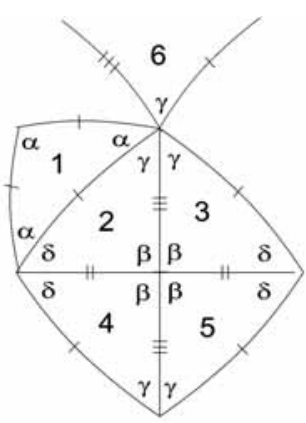

II

Fig. 16. - Planar representations.

Taking as starting point the configuration I, in Figure 16 we get the one in Figure 17-I, which is impossible to be expanded, since the alternate angle sums at vertex $v_{9}$ do not satisfy the angle folding relation ( $\delta$ is not a submultiple of $\pi$ and there are no other way of combining $\delta$ with anyone of the other angles).

The other possible position for tile 6 gives rise to configuration II. However, the alternate angle sum containing $\alpha, \gamma$ and $\delta$ at vertex $v_{10}$ do not satisfy the angle folding relation and so it is impossible to expand the configuration. 


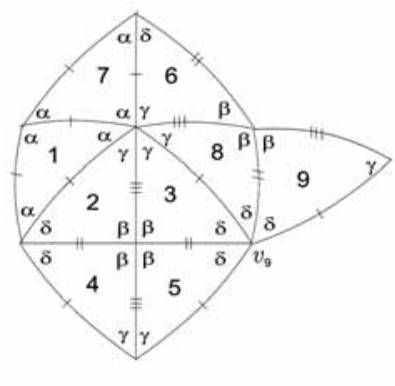

I

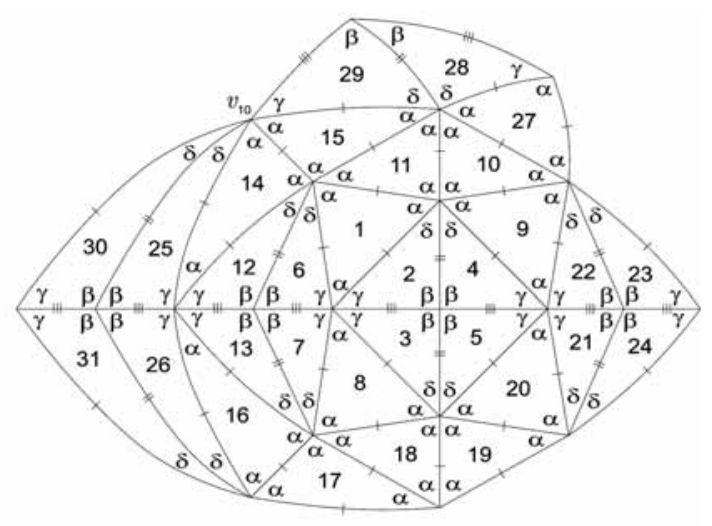

II

Fig. 17. - Planar representations.

2.3 Assume now that $\alpha+\gamma+t \delta=\pi, t \geq 1$. The discussion of the case $t>2$ follows a similar reasoning to the one done for $t=2$ and so we will focus our attention in the cases $t=1$ and $t=2$.

2.3.1 If $t=1$, the configuration illustrated in Figure 18, having as a starting point the one given in Figure 14 corresponds to a particular choice of the position of tiles 6,11 and 21 . It gives rise to a tiling $\tau \in \Omega\left(T_{1}, T_{2}\right)$ composed by 24 scalene triangles and 8 equilateral triangles and will be denoted by $\mathcal{F}_{1}^{\delta}$.
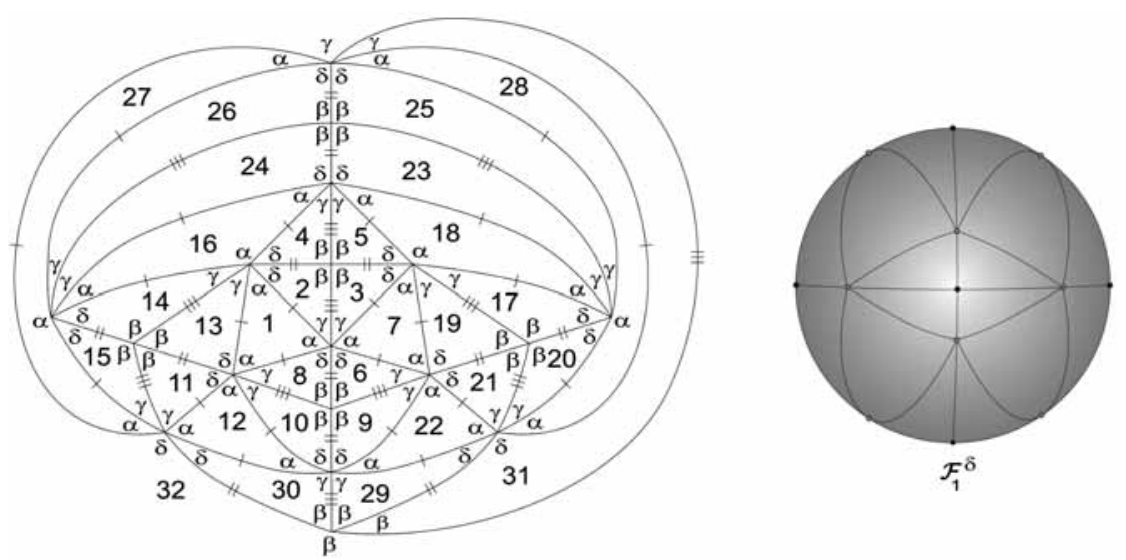

Fig. 18. $-2 \mathrm{D}$ and $3 \mathrm{D}$ representation of $\mathcal{F}_{1}^{\delta}$. 
The other possible arrangement for the length sides of tile 21 leads us to a global configuration of a tiling $\tau \in \Omega\left(T_{1}, T_{2}\right)$. This f-tiling is composed by 24 scalene triangles and 8 equilateral triangles and is denoted by $\mathcal{F}_{2}^{\delta}$, Figure 19.
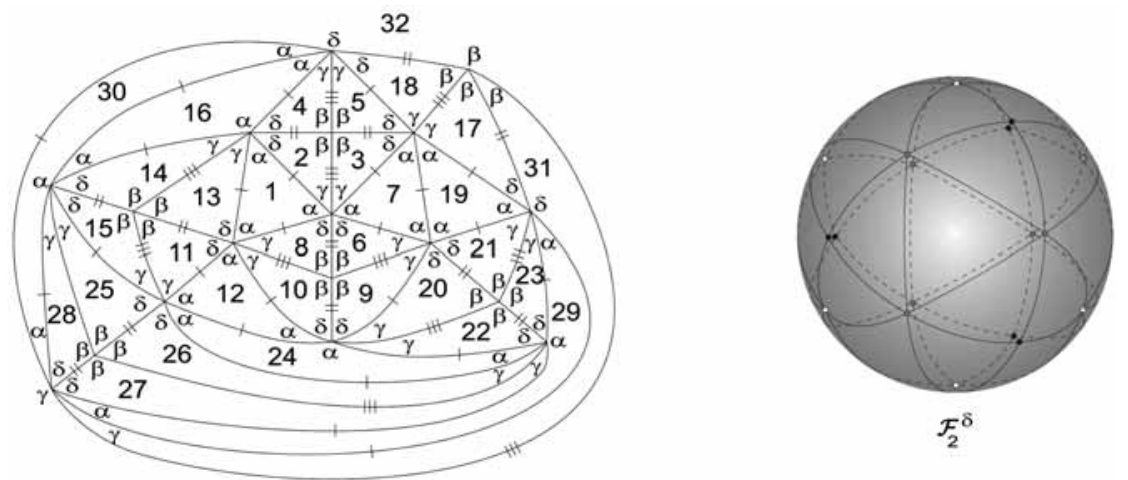

Fig. 19. $-2 \mathrm{D}$ and $3 \mathrm{D}$ representation of $\mathcal{F}_{2}^{\delta}$.

The other position of tile 11 leads to the configuration shown in Figure 20.

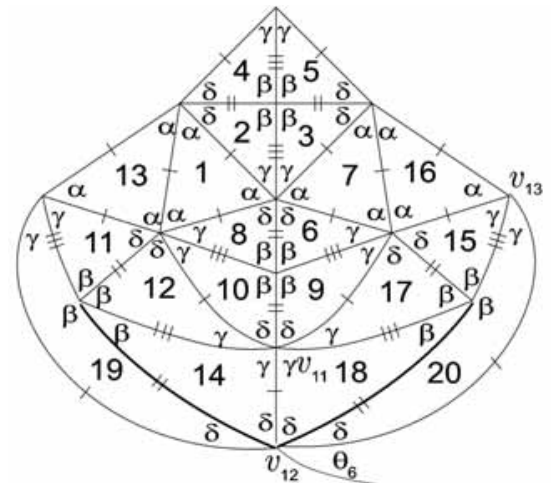

Fig. 20. - Planar representation.

Looking at vertex $v_{11}$, we conclude that $\gamma+\delta+\gamma=\pi$ (as $2 \gamma+\delta+\rho>\pi$, for any $\rho \in\{\alpha, \gamma, \delta, \beta\})$ and so $\alpha=\gamma$. At vertex $v_{12}$, the angle $\theta_{6} \in\{\delta, \gamma, \alpha\}$. 
2.3.1.1 If $\theta_{6}=\delta$, then at vertex $v_{13}$, we obtain the alternate angle sum $2 \gamma+\alpha \leq \pi$, which is an absurd.

2.3.1.2 If $\theta_{6}=\gamma, v_{12}$ is a vertex of valency greater than 6, otherwise $\alpha=\gamma=\delta$. Consequently, one of the alternate angle sums is $\gamma+q \delta=\pi, q \geq 3$. Since $2 \gamma+\delta=\pi$ and $\gamma+q \delta=\pi$ one has $\delta=\frac{\pi}{2 q-1} \alpha=\gamma=\frac{(q-1) \pi}{2 q-1}$. By the adjacency condition 1.1, we get $\frac{1+\cos ((q-1) \delta)}{\sin ((q-1) \delta)}=\frac{\cos \delta}{\sin \delta}$, that is, $\sin \delta=\sin ((q-2) \delta)$. Therefore, $q=3$ and as a result $\delta=\frac{\pi}{5}, \alpha=\gamma=\frac{2 \pi}{5}$.

However, extending configuration in Figure 20, we are led to an absurd, since the vertex surrounded by the angular sequence $(\gamma, \gamma, \gamma, \gamma, \alpha)$ violates the angle folding relation, see below (Figure 21).

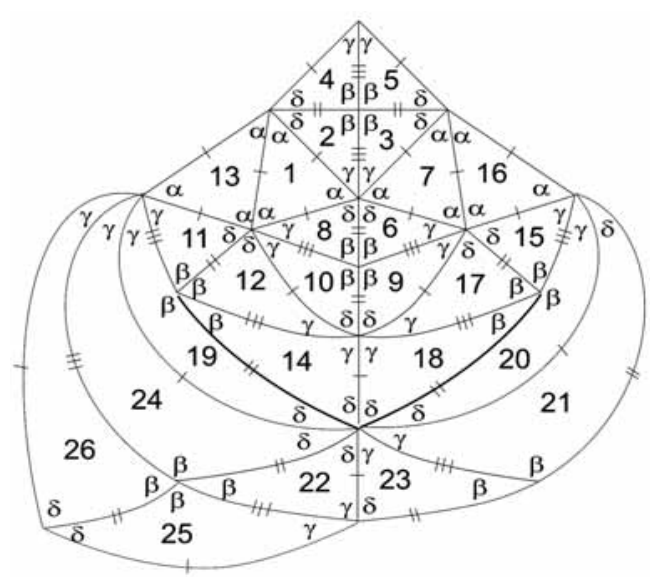

Fig. 21. - Planar representation.

2.3.1.3 If $\theta_{6}=\alpha$, since $\alpha=\gamma$, we get exactly the same absurd.

The other possibility for the length sides of tile 6 permits to extend a bit more the configuration in Figure 14 and a decision on $\theta_{7} \in\{\delta, \alpha\}$ must be taken (see Figure 22).

If $\theta_{7}=\delta$, the configuration is the one illustrated in Figure 23-I. This configuration expands globally and we get a representation of the tiling $\mathcal{F}_{2}^{\delta}$.

If $\theta_{7}=\alpha$, we get the configuration II illustrated in Figure 23. Observe that tile 16 obliges that one of the alternate angle sums at vertex $v_{14}$ is $2 \alpha+\delta=\pi$. Adding some new cells to this configuration we get a vertex 


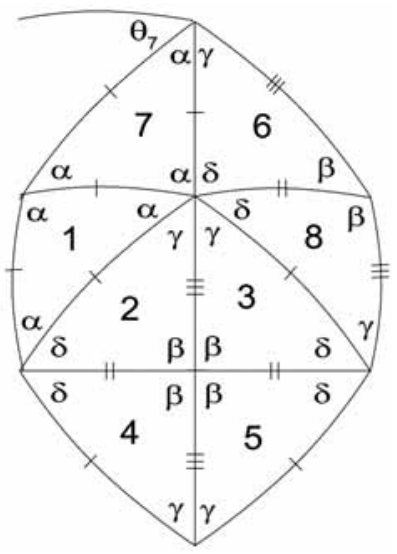

Fig. 22. - Planar representation.
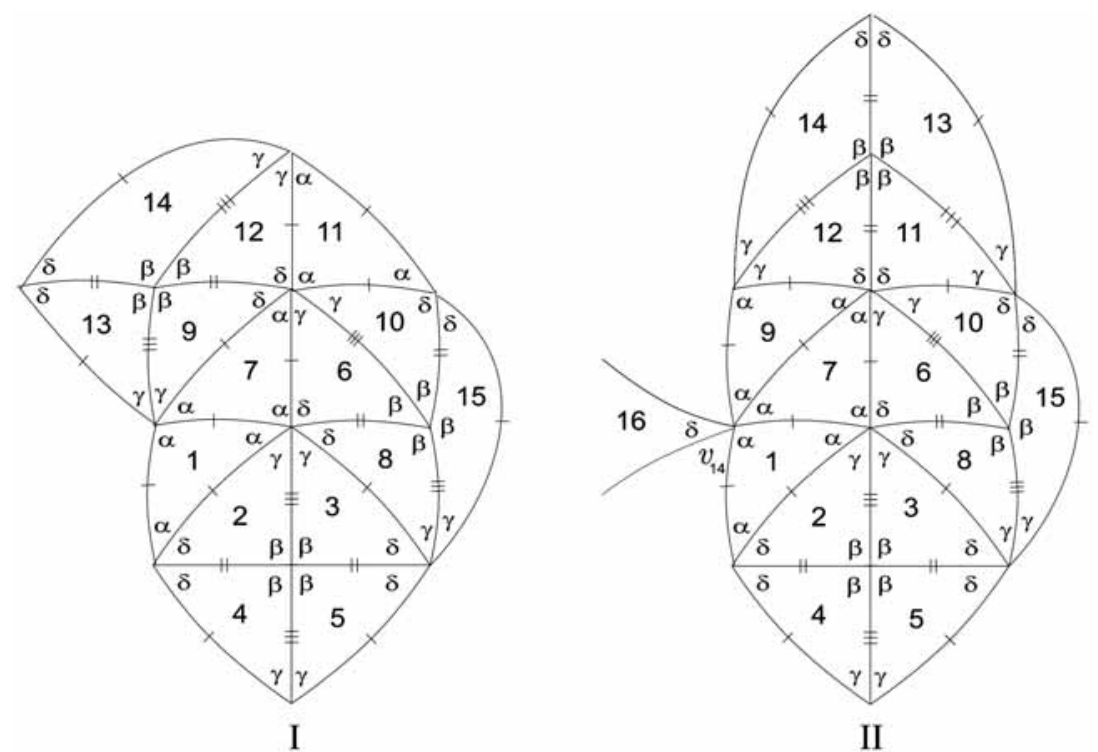

Fig. 23. - Planar representations.

surrounded by alternate angles $(\gamma, \gamma, \alpha, \gamma, \gamma)$, which is absurd (see Figure 24). Suppose now, that $\alpha+\gamma+t \delta=\pi$, for $t>1$. Having in account that $\gamma+\delta>\frac{\pi}{2}$, then $\gamma>\alpha+(t-2) \delta \geq \alpha$.

Summarizing, $\delta<\frac{\pi}{6}<\frac{\pi}{3}<\alpha<\gamma<\beta=\frac{\pi}{2}$. 


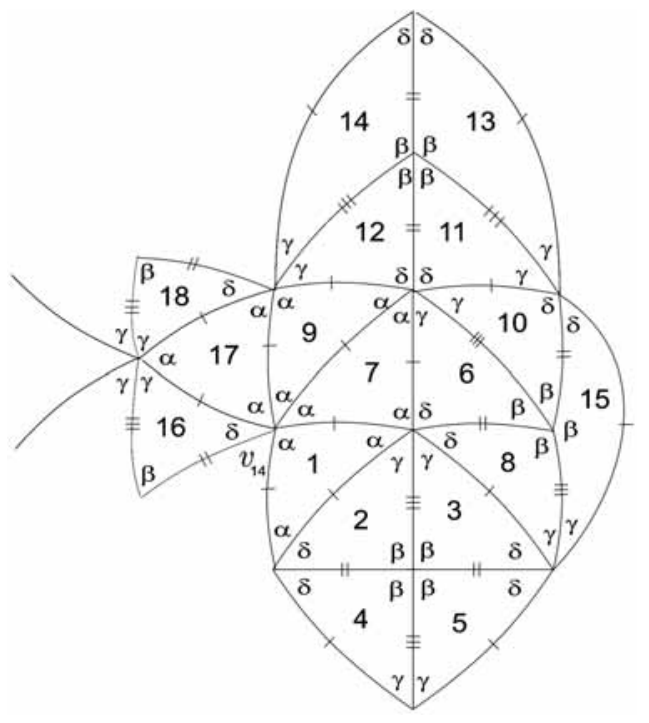

Fig. 24. - Planar representation.

\subsubsection{Suppose $t=2$.}

2.3.2.1 The configuration in Figure 25 corresponds to a choice for the positions of tiles 6 and 19, with $\theta_{8} \in\{\delta, \gamma, \alpha\}$.

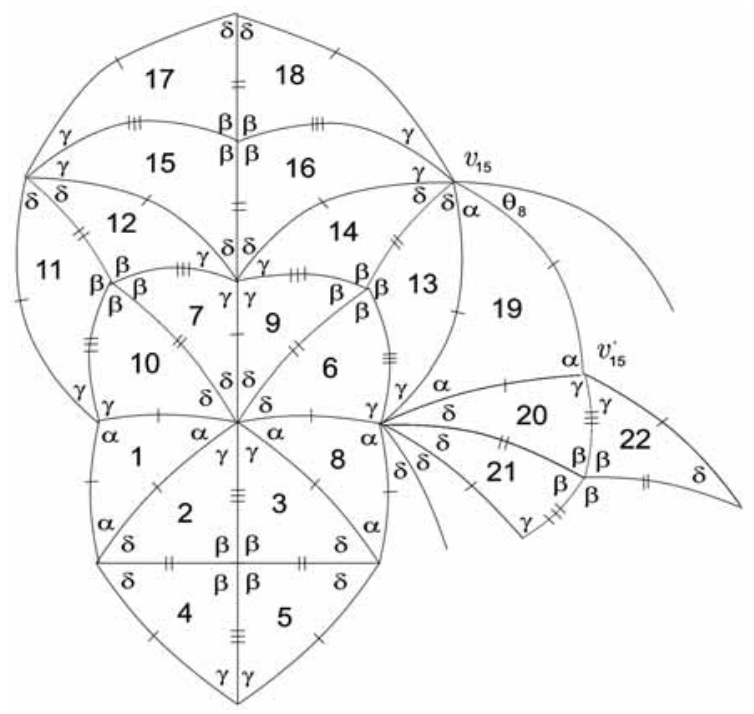

Fig. 25. - Planar representation. 
If $\theta_{8}=\delta$, then vertex $v_{15}^{\prime}$ is of valency 6 with alternate angle sums $2 \gamma+\delta=\pi$ and $\alpha+\delta+\gamma=\pi$, contradicting our assumption.

If $\theta_{8}=\gamma$, one of the alternate angle sum at vertex $v_{15}$ is $2 \gamma+\delta+\mu=\pi$, for $\mu \in\{\alpha, \delta, \gamma, \beta\}$, which is an absurd. Therefore, $\theta_{8}=\alpha$. Adding some new cells to the configuration we are led to the one in Figure 26, containing a vertex surrounded by the angular sequence $(\gamma, \gamma, \alpha, \gamma, \gamma, \ldots)$. Since $\gamma+\alpha+\gamma>\pi$, this configuration does not give rise to a f-tiling.

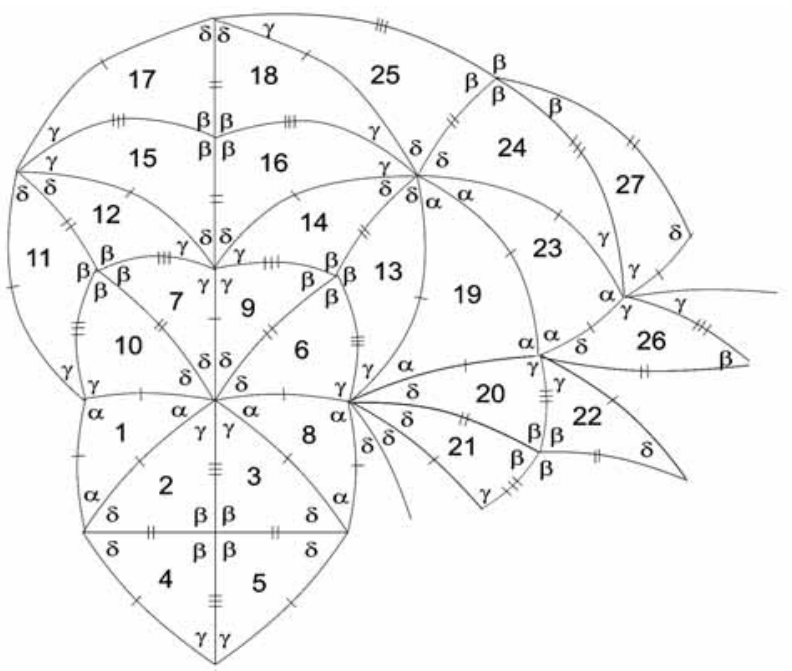

Fig. 26. - Planar representation.

2.3.2.2 If tiles 19 and 21 are, respectively, a scalene triangle and an equilateral triangle, then tile 27 is an equilateral triangle. We are led to a vertex surrounded by the angular sequence $(\gamma, \gamma, \alpha, \gamma, \gamma, \ldots)$, which, as seen before, is an absurd (see Figure 27).

2.3.2.3 If tile 21 is a scalene triangle, adding some tiles to the configuration we end up to the one illustrated in Figure 28. Vertex $v_{16}$ is surrounded in circular order by the sequence $(\gamma, \delta, \delta, \delta, \delta, \delta, \delta)$ and $\gamma+3 \delta=\pi$ implies that $\delta=\alpha>\frac{\pi}{3}$, which is an absurd and $\gamma+4 \delta=\pi$ implies that $\delta=\frac{\pi}{7}, \gamma=\frac{3 \pi}{7}$ and $\alpha=\frac{2 \pi}{7}$, which is also an absurd. 


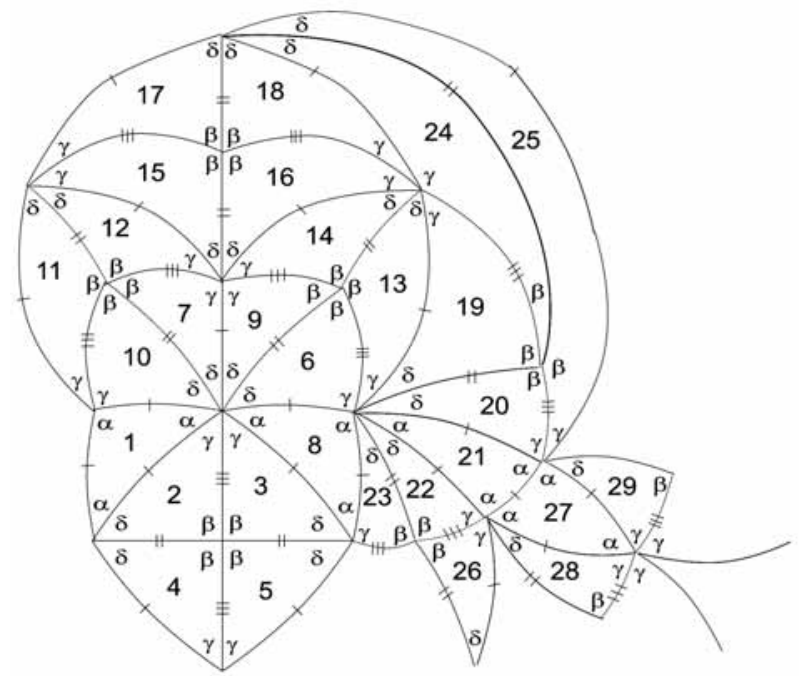

Fig. 27. - Planar representation.

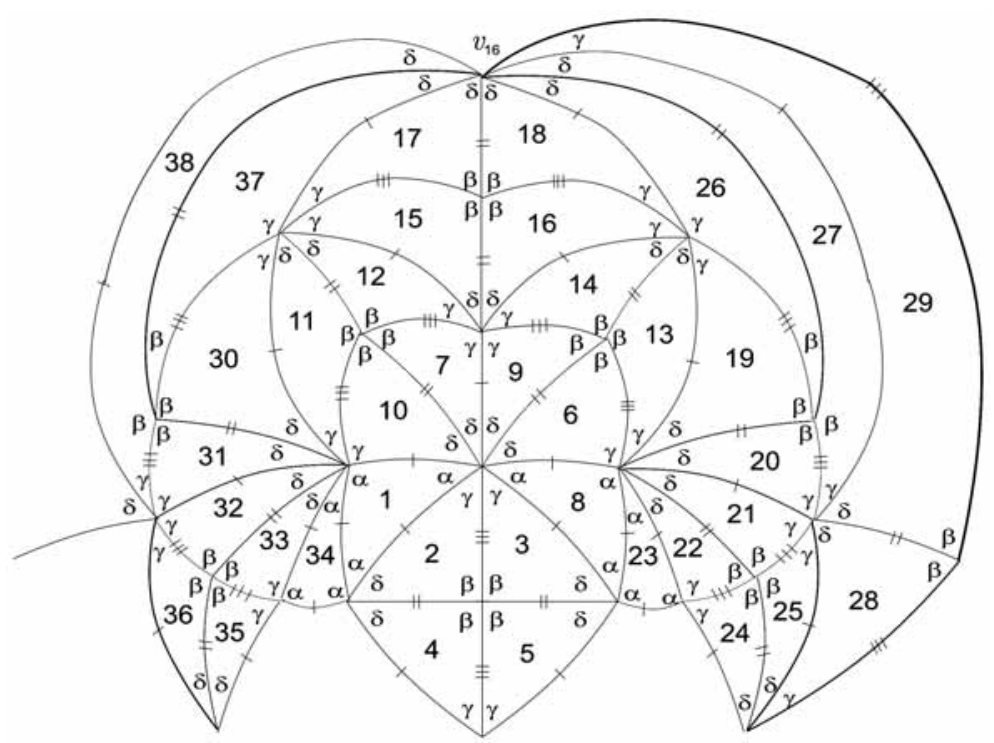

Fig. 28. - Planar representation.

2.3.2.4 Changing the order sides of tile 6 and assuming that tile 9 is a scalene triangle, we end up with configuration Figure 29, where $\theta_{9} \in\{\gamma, \alpha, \delta\}$. 


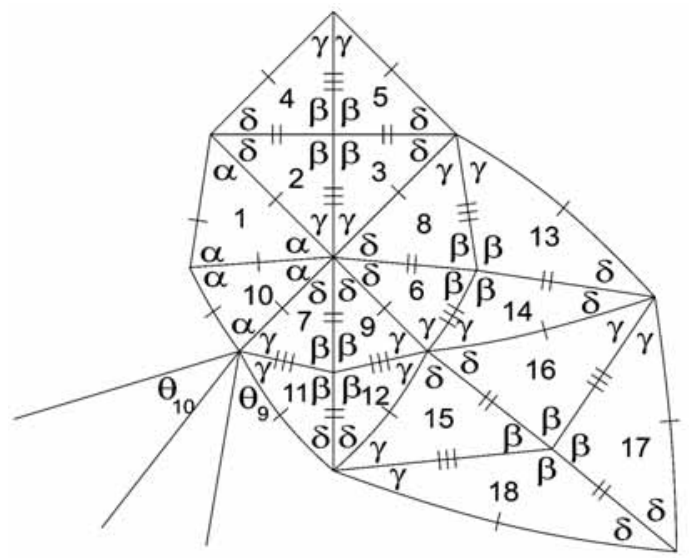

Fig. 29. - Planar representation.

If $\theta_{9}=\alpha$, we are led to a configuration with a vertex surrounded by alternate angles $\gamma, \delta, \delta, \delta$, which, as seen before, is an absurd. If $\theta_{9}=\gamma$, then one of the alternate angle sum at the vertex does not satisfy $\alpha+\gamma+2 \delta=\pi$. Since $\theta_{9}=\delta$, then $\theta_{10} \in\{\alpha, \delta\}$. Suppose $\theta_{10}=\alpha$. Then, extending the configuration we end up in an absurd similar to the one in 2.3.2.2. Therefore, $\theta_{10}=\delta$ and the configuration is the one illustrated below (Figure 30). At vertex $v_{17}$, one of the alternate angle sum is $\gamma+\alpha+\gamma>\pi$, which is an absurd.

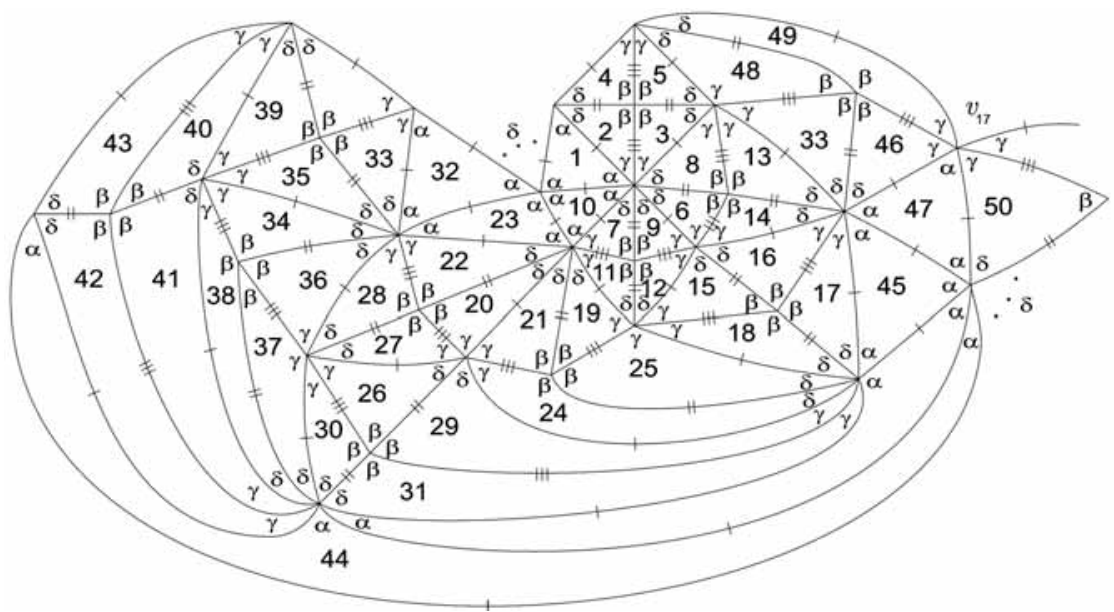

Fig. 30. - Planar representation. 
2.3.2.5 Finally, if tile 9 is an equilateral triangle, in order to have, at vertices $v_{18}$ and $v_{19}$, the alternate angle sum $\alpha+\gamma+2 \delta=\pi$, then $\theta_{11}=\alpha$ (see Figure 31).

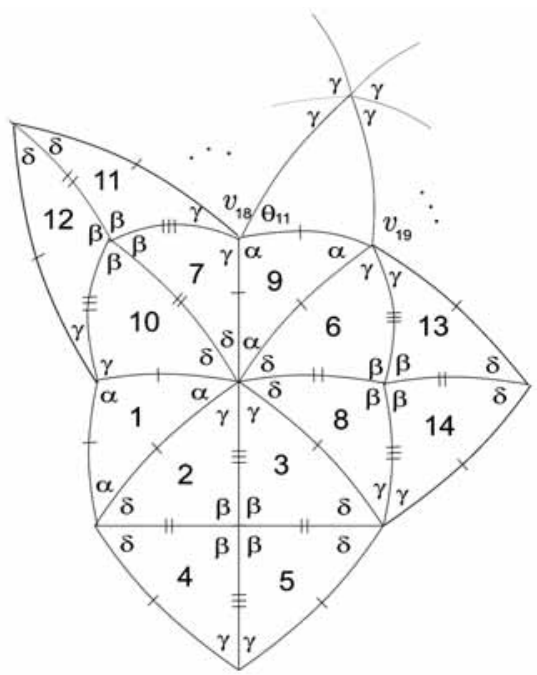

Fig. 31. - Planar representation.

Looking at vertex surrounded by angles $(\gamma, \gamma, \alpha, \gamma, \gamma)$, we conclude that this configuration will not expand, since $2 \gamma+\alpha>\pi$.

REMARK. - The equation (1.1) with $\beta=\frac{\pi}{2}$ and $\alpha=\pi-(\gamma+\delta)$ is writing in the form

$$
\frac{-\cos (\gamma+\delta)}{1+\cos (\gamma+\delta)}=\frac{\cos \gamma \cos \delta}{\sin \gamma \sin \delta}
$$

and one the angles $\gamma$ or $\delta$ is completely determined when the remaining is fixed.

In [3] it was proved that (2.1) has the solution $\delta=\gamma \approx 54.735^{\circ}$ (case when the triangle $T_{2}$ is isosceles). The graphic illustration in Figure 32 shows the variations of $\gamma$ and $\alpha=\pi-(\gamma+\delta)$, when $\delta \in] 0,54.735^{\circ}$ [. Therefore the f-tilings $\mathcal{F}_{1}^{\delta}$ and $\mathcal{F}_{2}^{\delta}$ represented in Figure 18 and Figure 19, respectively, are continuous families with $0<\delta<54.735^{\circ}$. 


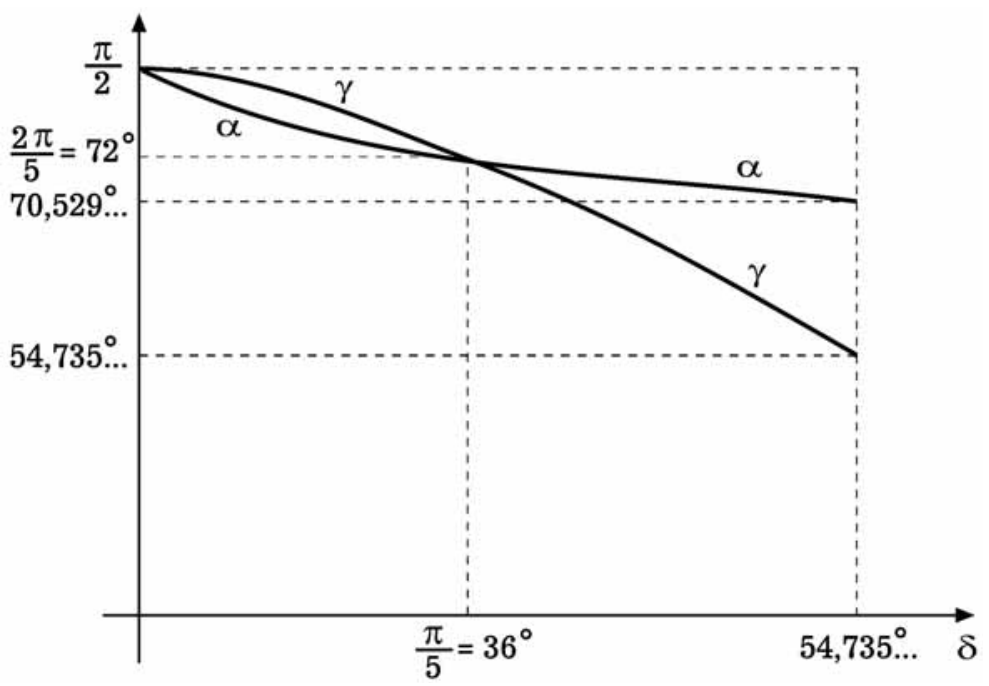

Fig. 32. $-\alpha$ and $\gamma$ as functions of $\delta$.

\section{Symmetry Groups}

Here we present the group of symmetries of the spherical f-tilings obtained $\mathcal{F}_{1}^{\delta}$ and $\mathcal{F}_{2}^{\delta}$. We also determine the transitivity classes of isogonality and isohedrality.

In Table 1 it is shown a list of the dihedral f-tilings, whose prototiles are an equilateral triangle $T_{1}$ of angle $\alpha$ and a scalene triangle $T_{2}$ of angles $\delta, \gamma$, $\beta,(\delta<\gamma<\beta)$ in a particular way of adjacency. We have used the following notation.

- $M$ and $N$ are, respectively, the number of triangles congruent to $T_{1}$ and the number of triangles congruent to $T_{2}$ used in such dihedral ftilings;

- $G(\tau)$ is the symmetry group of the f-tiling $\tau$. We shall say that the tiles $T$ and $T^{\prime}$ of $\tau$ are in the same transitivity class if the symmetry group $G(\tau)$ contains a transformation that maps $T$ into $T^{\prime}$. If all the tiles of $\tau$ form one transitivity class we say that $\tau$ is tiletransitive or isohedral. If there are $k$ transitivity classes of tiles, then $\tau$ is $k$-isohedral. On the other hand, if $G(\tau)$ contains transformations that map every vertex of $\tau$ into another vertex, then we say that the vertices form one transitivity class or that $\tau$ is $i s o-$ gonal. If there are $k$ transitivity classes of vertices, then $\tau$ is $k$ - 
isogonal. The numbers of isohedrality-classes and isogonalityclasses for the symmetry group are denoted, respectively, by \# isoh. and \# isog. .

With respect to tiling $\mathcal{F}_{1}^{\delta}$ the reflections on the coordinate planes generate $G\left(\mathcal{F}_{1}^{\delta}\right)$. It follows that $G\left(\mathcal{F}_{1}^{\delta}\right)$ is isomorphic to $C_{2} \times C_{2} \times C_{2}$. Now, there are 1 transitivity class of equilateral triangles and 3 transitivity classes of scalene triangles, which means that $\mathcal{F}_{1}^{\delta}$ is 4isohedral. On the other hand, numbering the vertices of the first octant, we conclude that $\mathcal{F}_{1}^{\delta}$ is 6 -isogonal.

Concerning to tiling $\mathcal{F}_{2}^{\delta}$, the rotation of an angle $\frac{2 \pi}{3}$ around the $x x$ axis generate $G\left(\mathcal{F}_{2}^{\delta}\right)$, and so it is a cyclic group. It follows that $\mathcal{F}_{2}^{\delta}$ is 12-isohedral (2 transitivity classes of equilateral triangles and 10 transitivity classes of scalene triangles) and 6-isogonal.

- The angles $\delta$ and $\gamma$ obey (2.1). Besides, $\alpha=\pi-(\gamma+\delta)$.

- $\delta_{0} \approx 54.735^{\circ}$ and $\gamma_{0} \approx 70.529^{\circ}$.

TABLE 1. -The Combinatorial Structure of the Dihedral F-Tilings of the Sphere by Equilateral and Scalene Triangles with adjacency of type I

\begin{tabular}{|c|c|c|c|c|c|c|c|c|c|}
\hline f-tilings & $\delta$ & $\gamma$ & $\alpha$ & $\beta$ & $M$ & $N$ & $G(\tau)$ & \# isoh. & \# isog. \\
\hline $\mathcal{F}_{1}^{\delta}$ & ] $0, \delta_{0}[$ & ]$\delta_{0}, \frac{\pi}{2}[$ & ]$\gamma_{0}, \frac{\pi}{2}[$ & $\frac{\pi}{2}$ & 8 & 24 & $C_{2} \times C_{2} \times C_{2}$ & 4 & 6 \\
\hline $\mathcal{F}_{2}^{\delta}$ & ] $0, \delta_{0}[$ & ]$\delta_{0}, \frac{\pi}{2}[$ & ]$\gamma_{0}, \frac{\pi}{2}[$ & $\frac{\pi}{2}$ & 8 & 24 & $C_{3}$ & 12 & 6 \\
\hline
\end{tabular}

\section{REFERENCES}

[1] Catarina P. Avelino - Altino F. Santos, Spherical F-Tilings by Triangles and $r$-Sided Regular Polygons, $r \geq 5$, Electronic Journal of Combinatorics, 15 (1) (2008), \#R22.

[2] A. M. D’Azevedo Breda, A Class of Tilings of $S^{2}$, Geometriae Dedicata, 44 (1992), pp. 241-253.

[3] A. M. D’Azevedo Breda - Patrícia S. Ribeiro - Altino F. Santos, A Class of Spherical Dihedral F-Tilings, European Journal of Combinatorics, 30 (1) (2009), pp. 119-132.

[4] A. M. D’Azevedo Breda - Patrícia S. Ribeiro - Altino F. Santos, Dihedral F-Tilings of the Sphere by Equilateral and Scalene Triangles-II, Electronic Journal of Combinatorics, 15 (1) (2008), \#R91.

[5] A. M. D’Azevedo Breda - Patrícia S. Ribeiro - Altino F. Santos, Dihedral F-Tilings of the Sphere by Equilateral and Scalene Triangles-III, Electronic Journal of Combinatorics, 15 (1) (2008), \#R147. 
[6] A. M. D’Azevedo Breda - Altino F. Santos, Dihedral F-Tilings of the Sphere by Spherical Triangles and Equiangular Well Centered Quadrangles, Beiträge Algebra Geometrie, 45 (2004), pp. 441-461.

[7] A. M. D'Azevedo BReda - A. F. SAntos, Dihedral F-Tilings of the Sphere by Rhombi and Triangles, Discrete Math. Theoretical Computer Sci., 7 (2005), pp. 123-140.

[8] M. Berger, Geometry, Volume 2, Section 18.4, Springer-Verlag, New York, 1996.

[9] S. A. Robertson, Isometric Folding of Riemannian Manifolds, Proc. Royal Soc. Edinb. Sect. A, 79 (1977), pp. 275-284.

Manoscritto pervenuto in redazione il 15 gennaio 2009. 